\title{
OPTIMIZATION OF THE SOUND OF ELECTRIC VEHICLES ACCORDING TO UNPLEASANTNESS AND DETECTABILITY
}

\author{
Petiot, Jean-François; Legeay, Killian; Lagrange, Mathieu \\ Ecole Centrale de Nantes, LS2N (UMR CNRS 6004)
}

\begin{abstract}
Electric Vehicles (EVs) are very quite at low speed, which can be hazardous for pedestrians. It is necessary to add warning sounds but this can represent an annoyance if they are poorly designed. On the other hand, they can be not enough detectable because of the masking effect due to the background noise. In this paper, we propose a method for the design of EV sounds that takes into account in the same time detectability and unpleasantness. It is based on user tests and implements Interactive Genetic Algorithms (IGA) for the optimization of the sounds. Synthesized EV sounds, based on additive synthesis and filtering, are proposed to a set of participants during a hearing test. An experimental protocol is proposed for the assessment of the detectability and the unpleasantness of the EV sounds. After the convergence of the method, sounds obtained with the IGA are compared to different sound design proposals. Results show that the quality of the sounds designed by the IGA method is significantly higher than the design proposals, validating the relevance of the approach.
\end{abstract}

Keywords: Optimisation, User centred design, Participatory design, Sound quality

\section{Contact:}

Petiot, Jean-François

Ecole Centrale de Nantes

LS2N

France

Jean-Francois.Petiot@ec-nantes.fr

Cite this article: Petiot, J.-F., Legeay, K., Lagrange, M. (2019) 'Optimization of the Sound of Electric Vehicles According to Unpleasantness and Detectability', in Proceedings of the 22nd International Conference on Engineering Design (ICED19), Delft, The Netherlands, 5-8 August 2019. DOI:10.1017/dsi.2019.402 


\section{INTRODUCTION}

Electric vehicles (EVs) and hybrid vehicles are becoming a serious alternative to internal combustion engine cars (Gillibrand et al., 2011). However, at low speed (under $40 \mathrm{~km} / \mathrm{h}$ ), EVs are very quiet and may be dangerous for pedestrian or visually impaired peoples who have to rely on auditory cues when intending to cross a road (Parizet et al., 2014). Regulation concerning the sounds of electric cars is still under study. Nevertheless, several countries such as Japan and the United States of America already decided that adding artificial sounds to EVs is compulsory (Konet et al., 2011). Car manufacturers started to design warning sound generator devices, named for instance AVAS (Approaching vehicle Audible System), VSP (Vehicle Sound for Pedestrian), VPNS (Vehicle Proximity Notification Systems)... Many studies have been conducted to recommend design guidance for warning sounds and external sound generation systems (Senselab, 2011), (Robart et al., 2013), (Singh et al., 2014).

The sonification of EVs is a complex design problem, with many constraints and stakeholders involved. Stakeholders include cyclists and pedestrians some of whom might have difficulties hearing warning sounds in a urban environment, drivers who expect audio-feedback on the performance of the car, and other third parties who prefer not to be disturbed by additional sounds (Petiot et al., 2013). The main difficulty in the design concerns the tradeoff between detectability and acceptance of the EV sound (Lee et al., 2017).

It is indeed clear that EV sounds may be masked by the background noise of the environment, making them hard to detect. And a naïve solution consisting in a simple increase of the sound level to reduce the masking effect may have dramatic consequences on the sound pollution of cities. There is then clearly a conflict between detectability and annoyance for the perception of EV sounds. Different studies addressed this problem (Campillo-Davo and Rassili, 2016), (Lee et al., 2017), (Parizet et al., 2014). All these studies are based on hearing tests of a predefined set of sound stimuli. They produced interesting results to give recommendations for the design of sounds by the fitting of a model between the perceptual dimensions and the sounds parameters. This data modeling stage may constitute a limitation for the optimization of sounds, given the number of parameters of the sounds and the possible interaction between them.

To address this problem, we propose in this paper an interactive optimization of EV sounds based on Interactive Genetic Algorithms (IGA). This model-free approach is efficient to improve design solutions during interactive assessments (Poirson et al., 2013), and to take into account subtle perceptual phenomenon (Wakefield et al., 2005). The first objective is to define a design method for the optimization of the tradeoff detectability/unpleasantness of EV sounds. It is based on hearing tests of synthesized EV sounds by a panel of participants, and uses Interactive Genetic Algorithms (IGA) for the optimization of quality (or fitness) of the sounds. A second objective of the paper is to assess the efficiency of the design solutions compared to different designs and current proposals of a designer.

The remainder of the paper is organized as follows. Section 2 presents a short background on interactive Genetic Algorithms (IGA) and their use in product design. Section 3 presents the material and methods for the experiment. It starts with a presentation of the sound synthesis method and the associated parameters, the tasks given to the participants, the sound stimuli used, and the method used for the analysis of the results. Results are presented in section 4. The concluding section provides implications for sound design and perspectives.

\section{BACKGROUND ON IGA}

\subsection{Principles}

Genetic Algorithms (GA) are evolutionary optimization methods (Goldberg, 1989) The principle of GA is based on iterative generations of population of individuals, converging step by step toward solutions, which are adapted to the problem. Based on the principle of Darwin's natural evolution theory, the algorithm proceeds to a selection of parents, which will spread their genetic dominant heritage in the next generation, suitable to a desired objective. Classically, the fitness evaluation of the individuals is calculated numerically with a mathematical function known beforehand. A particular category of GA, Interactive Genetic Algorithms (IGA) introduces the user in the optimization loop to assess the fitness. During each iteration the user selects solutions (products) that he/she considers as the most interesting for the desired objective. After a number of iterations, the method may converge 
towards solutions that fulfill the user's objective. These algorithms are used for example to explore design spaces and to encourage creativity (Kim and Cho, 2006), (Qian and Ben-Arieh, 2009). Since the user decides the individual fitness, there is no need for a prior and unique formulation of the fitness function. For some applications, such as exploring semantic dimensions (Poirson et al., 2013) or integrating complex perceptual processes (Wakefield et al., 2005) (Lee and Chang, 2010), this advantage is crucial.

\subsection{Implementation of IGA}

After a definition of the variables of the product and their corresponding levels, a coding of the designs, represented by a chromosome, is proposed. Our implementation uses a binary coding and discrete-valued variables. A more complete description of the implementation of our IGA can be found in (Poirson et al., 2013). The IGA creates an initial population of designs by randomly generating the chromosomes and presents them to the user (e.g. pictures or sounds). Based on personal criteria, and according to the instructions given to the user for the experiment, the user has to select a subset of these individuals ( 1 or 2 ), or to rate each individual according to its "fitness". A new population of individuals is then created using one of three different operations on each individual from the previous population. The efficiency of our IGA is ruled by its 3 operators: crossover, mutation, and selection, chosen randomly for each individual of the population, and controlled by the crossover rate $\left(c_{r}\right)$, the mutation rate $\left(m_{r}\right)$ and the selection rate $\left(s_{r}\right)$. These values are chosen between 0 and 1 in such a way that $c_{r}+m_{r}+s_{r}=1$. An indicator, rand(i), is randomly chosen between 0 and 1 for each individual $i$ with a uniform distribution.

- If $\operatorname{rand}(\mathrm{i})<c_{r}$, the operation is a crossover (single point crossover - the second parent is chosen randomly in the population)

- If $c_{r} \leq \operatorname{rand}(\mathrm{i}) \leq c_{r}+m_{r}$, the operation is a mutation (random mutation of one variable)

- If rand(i) $>c_{r}+m_{r}$, the operation is a selection (simple duplication of the individual)

A fourth important parameter of the IGA is the roulette wheel $w_{r}$. In the crossover operation, the probability that an individual will be a parent in the crossover operation is increased by the weight $w_{r}>1$. An automatic process was implemented to tune the different parameters of our IGA (Poirson $e t$ al., 2013). This process uses simulated "virtual" users and a "target" product in the design space (defined by target values of the design variables). To simulate the choices of a virtual user, a distance function between the individuals of the population and the target is computed. By launching several simulations in the same conditions (Monte Carlo method), an average estimate of the convergence rates of the IGA is computed, given the value of the parameters. This process allows the experimenter to determine the "optimal" tuning of the parameters, given a maximum number of generations.

\section{MATERIAL AND METHODS}

\subsection{EV sound synthesis}

\subsubsection{Components of the sound}

The EV sounds were synthesized using the mathematical modeling software Matlab and the additive synthesis technique. In order to generate different but plausible sounds for an electric car, after an analysis of current sounds of different carmakers (Misdariis et al., 2012) and personal propositions (Petiot et al, 2013), four main components of the sound were considered. The components are also named design factors in this paper.

- Component C1 "A thermic motor sound". This component synthesizes the first harmonics of a classical 4-stroke internal combustion engine (H0.5, H1, H1.5, H2, H4, H6),

- Component C2 "A Harmonic Sound". This component synthesizes different musical 'notes', harmonic, that constitute a chord (chord with 2, 3, or 4 notes),

- Component C3 and C4: "A broad band Noise" (granular synthesis). These components synthesize two filtered noises.

The final temporal signal $s(t)$ is simply a weighted sum of the different components (equation 1).

$$
s(t)=a_{C 1} \cdot C 1(t)+a_{C 2} \cdot C 2(t)+a_{C 3} \cdot C 3(t)+a_{C 4} \cdot C 4(t)
$$


In addition to this, different parametric filters were applied to the final sound: (1) a sweeping filter (envelop filter), which changes the harmonics amplitude in function of time - (2) a flanging filter (swept comb filter effect), which produces time variations of the frequency spectrum.

Since it is out of the scope of this paper to describe all the parameters of the synthesizer (there are more than 70 independent parameters to define a sound), we can mention that all the frequencies and amplitudes of the components are adjustable, to create credible and original sounds, as well as the filters parameters. The sound is not constant but 'played' by a control parameter of the car: the speed. To make the sound evolve with the speed of the car, we choose to adjust the frequencies and the amplitudes of the different components according to the speed with parameterized patterns.

\subsubsection{Design variables of the EV sound}

Among the different synthesis parameters of the sounds, it is necessary to define which one are manipulated by the IGA and coded in the genome (space of exploration of the genetic code). After several experiments, the following 6 variables, and their corresponding levels, were chosen as factors to get a large diversity of sounds (table 1). The four first factors $(\mathbf{C 1}, \mathbf{C 2}, \mathbf{C 3}, \mathbf{C 4})$ control the frequencies of the components C1, C2, C3, C4, the factor Amp control the relative amplitude of the different components, and the Filter controls the use of different filters to alter the global sound. 4 levels were chosen for each factor. The setting of the levels of the factors required many adjustments (not reported here) to obtain audible differences between sounds, but with still convenient sounds.

Table 1. Definition of the 6 factors (design variables) and their levels

\begin{tabular}{|l|l|l|l|l|l|}
\hline Factor & Variable & Level 1 & Level 2 & Level 3 & Level 4 \\
\hline C1 & $\begin{array}{l}\text { Fundamental } \\
\text { frequency of C1 }\end{array}$ & $70 \mathrm{~Hz}$ & $100 \mathrm{~Hz}$ & $130 \mathrm{~Hz}$ & $160 \mathrm{~Hz}$ \\
\hline $\mathbf{C 2}$ & $\begin{array}{l}\text { Fundamental } \\
\text { frequency of C2 }\end{array}$ & $100 \mathrm{~Hz}$ & $150 \mathrm{~Hz}$ & $200 \mathrm{~Hz}$ & $250 \mathrm{~Hz}$ \\
\hline C3 & $\begin{array}{l}\text { Central frequency of } \\
\text { C3 }\end{array}$ & $100 \mathrm{~Hz}$ & $200 \mathrm{~Hz}$ & $300 \mathrm{~Hz}$ & $400 \mathrm{~Hz}$ \\
\hline C4 & Central frequency of & $500 \mathrm{~Hz}$ & $600 \mathrm{~Hz}$ & $700 \mathrm{~Hz}$ & $800 \mathrm{~Hz}$ \\
\hline Amp & Amplitude of C1, C2, & $\begin{array}{l}a_{C 1}=2 \\
a_{C 2}=1 \\
a_{C 3}=0.75 \\
a_{C 4}=0\end{array}$ & $\begin{array}{c}a_{C 1}=0.5 \\
a_{C 2}=0.75 \\
a_{C 3}=1.5 \\
a_{C 4}=0.33\end{array}$ & $\begin{array}{c}a_{C 1}=0.25 \\
a_{C 2}=0.5 \\
a_{C 3}=0.1 \\
a_{C 4}=0.25\end{array}$ & $\begin{array}{c}a_{C 1}=0 \\
a_{C 2}=0.1 \\
a_{C 3}=0.1 \\
a_{C 4}=0.5\end{array}$ \\
\hline Filter & Type of filter & None & Sweeping filter & Flanger & Sweep+flanger \\
\hline
\end{tabular}

With these six factors and four level, the design space counts $4^{6}=4096$ possible designs (all the possible combinations of the full factorial).

\subsection{Scenario and sound stimuli}

Given that the objective of the test is to assess the reaction time associated to the detection of the EV sound, the sound must be incorporated in a background noise (masking signal). The background noise considered in the study corresponds to a two-lane street in downtown. The soundscape was synthesized with the SimScene software ${ }^{1}$ (Rossignol et al., 2015), from real recordings in a city. To be used as background noise, the soundscape must not contain any strong emergent event (horns, car passing, ...) (Kerber and Fastl, 2008). The level of the background noise was adjusted to a convenient level and kept constant for all the stimuli proposed in the hearing test. To avoid the potential fatigue of the participant due to the repetition of the same background noise during the test, the part of the audio file selected (15 seconds) was randomly chosen in the total background noise (duration $1 \mathrm{mn}$ ). The scenario chosen for the test corresponds to the following situation (Misdariis et al., 2013): a pedestrian located on the sidewalk of a street waits before crossing (Figure 1). An EV may pass by, coming either from the right or from the left. The listener is static, and must indicate when he/she detects the EV.

\footnotetext{
${ }^{1}$ Open-source project available at: https://bitbucket.org/ mlagrange/simscene
} 


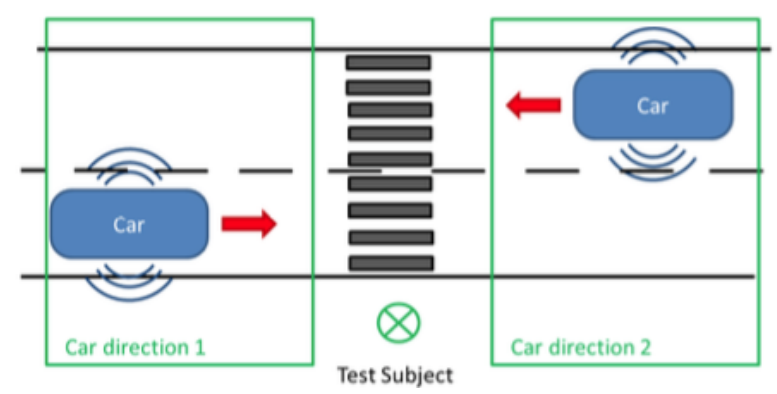

Figure 1. Passing-by scenario for the hearing test: pedestrian located on the sidewalk of a street

To obtain a pseudo-realistic passing-by scenario, the following properties have been implemented:

- The sound level of the EV is modulated according to the vehicle/listener distance. The model used, based on acoustic theory, considers the EV as a monopole and provides a sound level inversely proportional to the distance to the listener (1/r) (see figure 2) (Misdariis et al., 2013), (Lee et al., 2017).

- The speed of the EV is considered as constant and equal to $30 \mathrm{~km} / \mathrm{h}$

- The duration of the sound stimuli is 15 seconds

- The Doppler effect (shifting in frequency due to the speed of the source) is taken into account for a more realistic experience,

- The direction of the car (from the right or from the left) is randomly chosen

- The panning of the EV sound is managed in such a way that the source goes progressively from one canal (left or right, depending of the direction of the EV) to the other (right or left) according to the position of the vehicle

Figure 2 describes the assembly of the background and the EV sounds and their respective sound level evolution.

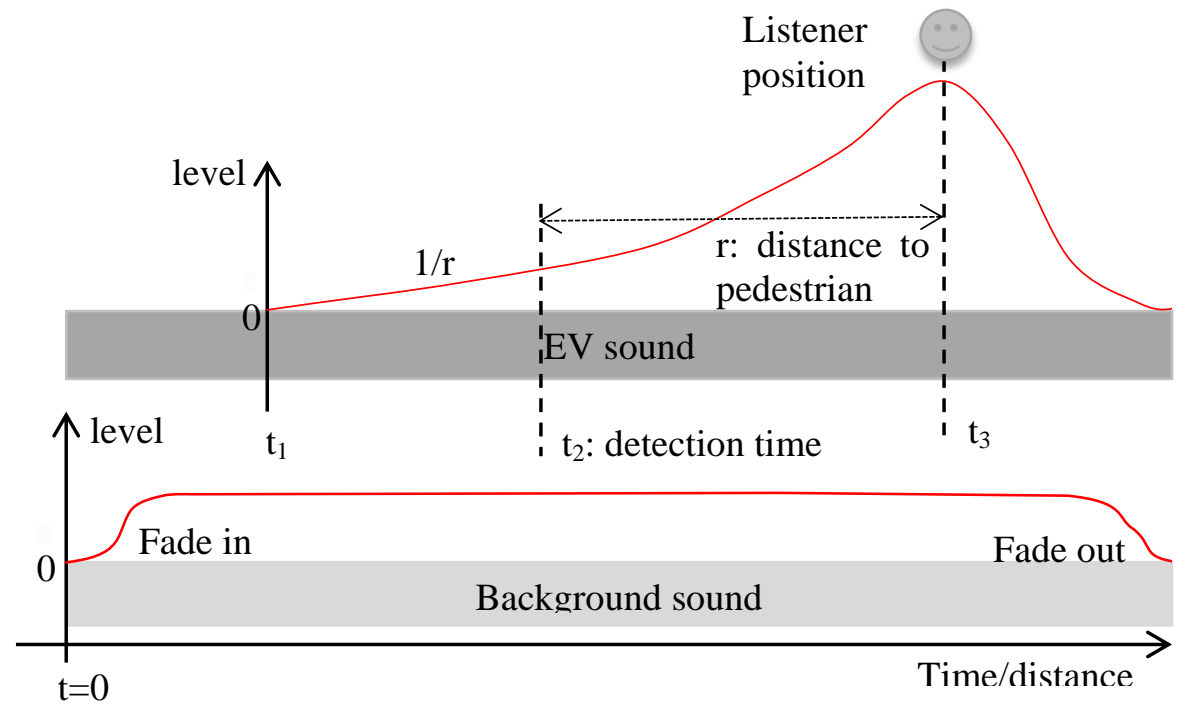

Figure 2. Timeline of the assembly of the background and the EV sound, with their respective level evolution (the $x$ axis represents indifferently the time or the distance of the EV, given that the speed of the vehicle is constant)

We can mention that for experimental reasons related to the test duration, the attenuation function of the EV sound is asymmetrical, as in (Misdariis et al., 2013) (the attenuation is more fast than the increase of the sound level). Of course this aspect does not affect the detectability of the EV sound, which always occurs in the approach phase.

\subsection{Experiment}

The objective of the experiment is to assess the detectability and the unpleasantness of EV sounds. 
15 participants (12 males), with no reported auditory deficiencies, performed the experiments. The audio stimuli were presented with the same hardware desktop configuration, sound card and software, as well as Beyerdynamics DT-990 headphones in a quiet environment. The same sound output level was set by the experimenter for all computers. Instructions were given to the participants at the beginning of the experiment, mainly to explain how to assess the detection time.

After selecting a sound ("select" button Figure 3), participants had to strike the "space bar" to start playing the stimuli $(\mathrm{t}=0)$, and next strike the "a" key as soon as they detect the EV coming from the left, or the "e" key if it is coming from the right. This allows the definition of the detection time $t_{2}$. The detection duration $D_{d}$ is then given by (equation 2).

$$
D_{d}=t_{2}-t_{1}
$$

In case the participant did not strike any key, or strike a key before $t_{1}$ (starting time of the EV), the detection duration was arbitrarily set to the maximum value, and a warning was recorded. In case the participant struck the wrong key (mistake in the direction of the EV), the detection duration was still computed, but a warning was recorded. The change in the direction of the car in the protocol is very important to be able to detect "false alarm" cases, where the participants strike the key before detecting the car. Furthermore, to avoid habituation of the participant in the detection time, the starting time $t_{1}$ of the EV sound in the mixture (figure 2) was not always the same, and was randomly chosen in the interval $[1,3]$ seconds.

After assessing the detectability of the sound, participants were asked to rate the unpleasantness $U n_{s}$ of the sound on a structured semantic scale (figure 3 - from " 0 ": not at all unpleasant to "10": very unpleasant)). To explain this semantic dimension unpleasantness, the following information was given to the participants « If the car passed by your house during a calm moment, how unpleasant would the sound be? ». They were able to play again the stimuli, but of course it was not possible to assess again the detectability (given that they already heard the sound and knew the direction of the car).

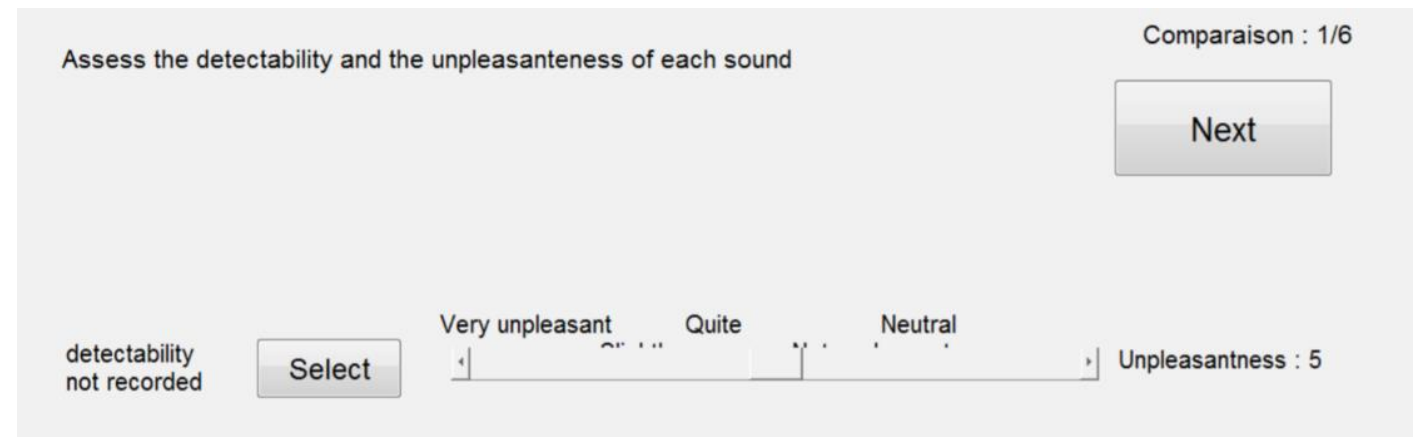

Figure 3. Interface for the assessment of the detectability and the unpleasantness of a sound stimulus (structured rating scale).

\subsection{IGA test}

The assessment of the EV sound according to detectability and unpleasantness has been included in an IGA iterative experiment. The problem of IGA tests is to manage a good balance between convergence properties and fatigue of the participant. After different tests, we considered that the assessment of 11 populations of 9 sounds was enough to not fatigue the participant. This corresponds to a test duration of around $35 \mathrm{mn}$. An automatic process was implemented to tune the different parameters of our IGA (Poirson et al., 2010). The optimal tuning parameters of the IGA are as follows:

- Wheelrate: $w_{r}=14$

- Crossrate: $c_{r}=0.65$

- $\quad$ Mutation rate: $m_{r}=0.3$

The fitness of the individuals was computed from the detectability and unpleasantness assessments (equation 3). The following form was defined, according to the detection duration $D_{d}$ and the unpleasantness rating score $U n_{s}$ :

$$
\text { fitness }=a\left(1-\frac{D_{d}}{t_{3}-t_{1}}\right)+\frac{1}{2}\left(10-U n_{s}\right)
$$


The higher the unpleasantness, and the higher the detection duration, the lower the fitness. The parameter $a$ represents the relative contribution of the detection time to the fitness. After several tests, it was set to $a=5$ to give a similar importance to detectability and unpleasantness given the variance in the population. At the end of the IGA test, the individual with the highest fitness $I G A_{\text {opt }}$ in the last population can be defined for each participant.

\subsection{Assessment of sounds proposals}

At the end of the IGA test, participants were asked to assess the detectability and unpleasantness of six different EV sound proposals (interface given in Figure 3). Four EV Sounds ( rand1, rand 2 , rand 3 , rand 4 )were randomly defined in the experimental space, whereas two EV sounds (Sound1,Sound 2) were designed by a sound designer with instructions for good detectability and good acceptance. In total, each participant had to assess 105 sound stimuli $(11 * 9$ for the IGA +6 design proposals). For each participant, the detection time, unpleasantness, fitness of his/her best individual $I G A_{o p t}$ was next compared to those of the different sound proposals. In addition to this, the detection time was converted into the distance to pedestrian, i.e. the distance of the EV to the participant at the instant of detection.

\section{RESULTS}

Concerning the detection of the car direction, one participant made more than $50 \%$ or errors in the detection direction (problem with the headphone). He was withdrawn of the panel. The other 14 participants made very few errors (less than 5 errors for the 105 stimuli), errors only in the direction of the car due to careless mistakes (strike of the wrong key). Their data were considered as valid.

\subsection{IGA convergence}

The average fitness of the population according to the generations for the whole panel of participants is presented in Figure 4.

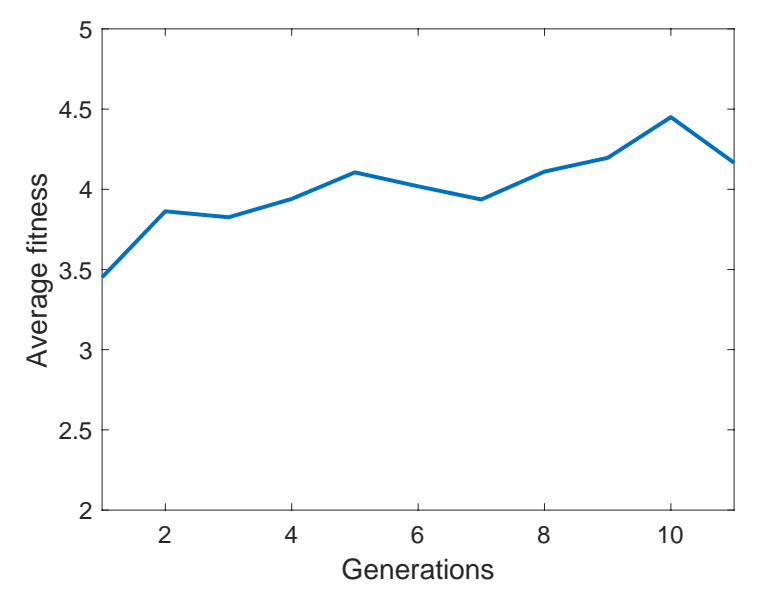

Figure 4. Average fitness of the populations over the generations.

An improvement of the fitness is noticed over the generations, sign of the reliability of the experimental protocol for the assessment of the detection time and the unpleasantness, and a correct tuning of the IGA parameters.

\subsection{Analysis of the best IGA sounds}

Table 2 shows the occurrences of each level of the variables in the set of $I G A_{o p t}$ sounds. For example, for the fundamental frequency of the motor sound (C1), 6 participants chose the level $3(130 \mathrm{~Hz}), 3$ the level $2(100 \mathrm{~Hz})$ or level $4(160 \mathrm{~Hz})$, and 2 the level $1(70 \mathrm{~Hz})$ for their final sound. To define the variables subjected to the most consensual choice concerning their levels, a multinomial goodness of fit test of the distribution of the occurrences was carried out. Results are presented in Table 2. 
Table 2: Occurrences of the levels of the variables in the IGA final choices of the participants (IGA test)

\begin{tabular}{|l|l|l|l|l|l|l|}
\hline & C1 & C2 & C3 & C4 & Amp & Filter \\
\hline Level 1 & 2 & 2 & 3 & 5 & 8 & 1 \\
\hline Level 2 & 3 & 4 & 4 & 4 & 0 & 4 \\
\hline Level 3 & 6 & 3 & 3 & 2 & 3 & 3 \\
\hline Level 4 & 3 & 5 & 4 & 2 & 2 & 5 \\
\hline $\begin{array}{l}\text { Multinomial } \\
\text { test Signif. }\end{array}$ & N.S. & N.S. & N.S. & N.S. & $* * *$ & N.S. \\
\hline & $* * *: p<0.01$ & & & \\
\hline
\end{tabular}

Only one variable (Amp) obtains occurrences significantly different from a random distribution at the $1 \%$ level. For the amplitude of the components of the sounds, the level 1 (strong presence of the thermic motor sound) is by far the most represented. This over-representation could be explained by the "naturalness" of the thermic motor sound in the street, and the habit of people to be surrounded by such noises.

In conclusion, for the whole group, the presence of the thermic motor sound is important for the detectability and the acceptance of the EV sounds. For the other variables $(\mathbf{C 1}, \mathbf{C 2}, \mathbf{C 3}, \mathbf{C 4}$, Filter), there was no significant consensus, mainly because of the small size of the panel of participants. Additional studies are needed to identify the sound that better fits in average the requirement of all participants.

\subsection{Comparison IGA sounds/design proposals}

Figure 5 presents, for the panel of 14 participants, the average performances of the sounds proposals and of the best sound $I G A_{o p t}$ according to the two criteria detection time and unpleasantness. The standard error is also reported for information.

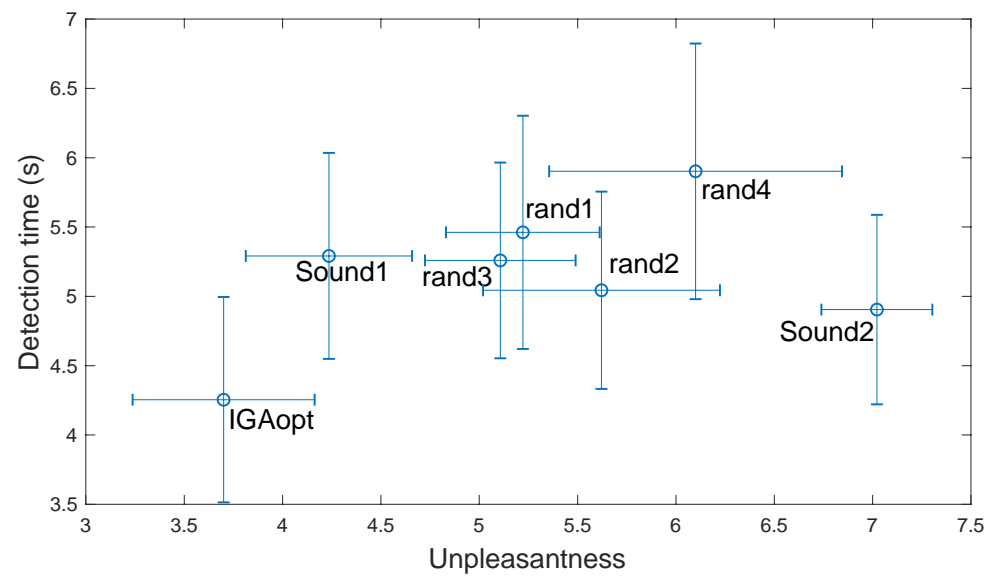

Figure 5. Scatterplot of the average performances of the different EV sounds (detection time vs. unpleasantness) with their standard error.

It is clear on Figure 5 that the $I G A_{o p t}$ sound is Pareto efficient. In average, the $I G A_{o p t}$ sound obtains the best performances both for detectability and unpleasantness. The first proposal of the sound designer, Sound1, is still not unpleasant, but less detectable than $I G A_{\text {opt }}$. The second proposal, Sound2, is more detectable, but the price to pay is the unpleasantness, that is the largest. The randomly generated sounds (randl to rand4) obtain average performances: they are rather unpleasant, and with large detection times.

To investigate the differences in the performances of the sounds, the average scores with their standard errors are presented in Figure 6 left (Detection time), Figure 6 right (distance to pedestrian), Figure 7 left (Unpleasantness), and Figure 7 right (Fitness). To study the differences in the average score, a one-tailed paired t-test is carried out for every pair of sounds (significant threshold: $p=0.05$ ). The results are presented with bold lines connecting the sounds in the figures. When sounds are connected, pairs are not significantly different, whereas they are when not connected. 
According to detection time, there is no significant difference between two groups of sounds (Figure 6 left): (rand2, Sound2, IGAopt) and (rand4, rand1, Sound1, rand3, rand2, Sound2). With detection time only, the $I G A_{\text {opt }}$ sound does not outrank two other proposals, particularly the sound designer proposal Sound2. Concerning the distance to pedestrian, figure 6 (right) shows that some proposals (rand4, randl) are detected under the stopping distance of the vehicle (considered as $11 \mathrm{~m}$ at $30 \mathrm{~km} / \mathrm{h}$ ). These sounds do not allow the EV to stay in a safety zone with regard to a blind detectability.
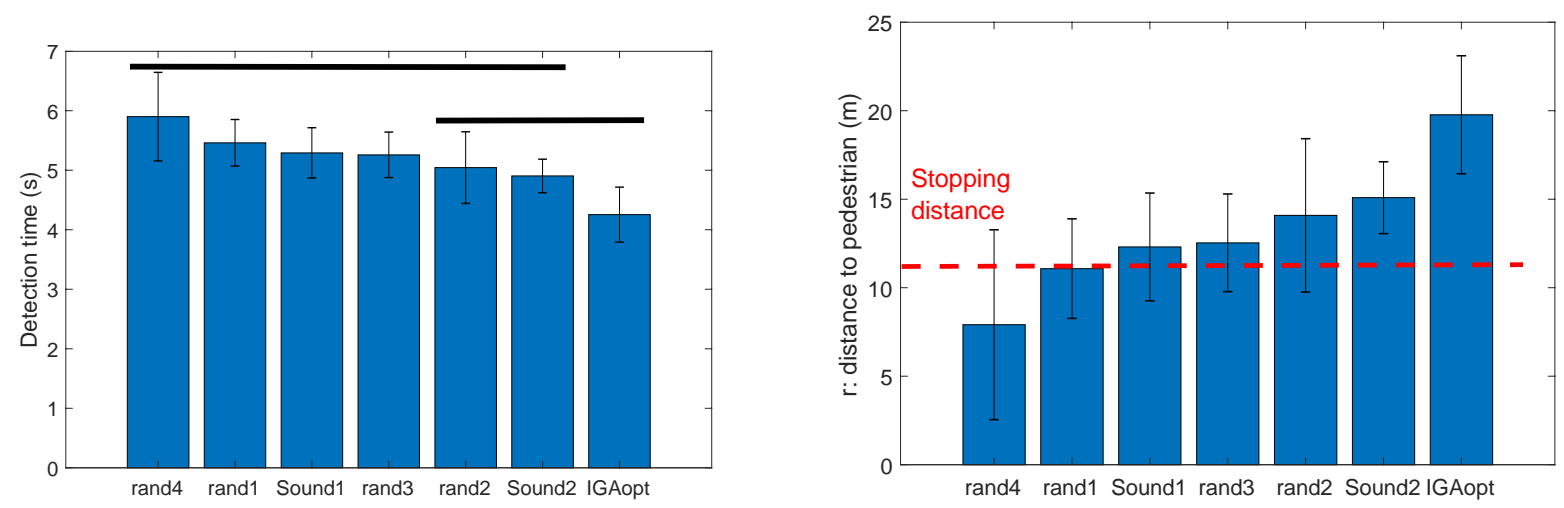

Figure 6. Bar graph of the average value of the Detection time (left) and distance to pedestrian (right) for the different EV sounds. Non significant differences between pairs of sounds ( $p>.05)$ are linked with an horizontal line (paired $t$-test).

According to Unpleasantness (Figure 7 left), there is no significant difference between three groups of sounds. Again the $I G A_{o p t}$ sound does not outrank all the proposals, particularly the sound designer proposal Soundl. When global fitness is considered (Figure 7 right), IGA $A_{o p t}$ is then significantly different of all the other proposals. This result shows that the IGA allows a conjoint optimization of the two conflicting criteria.
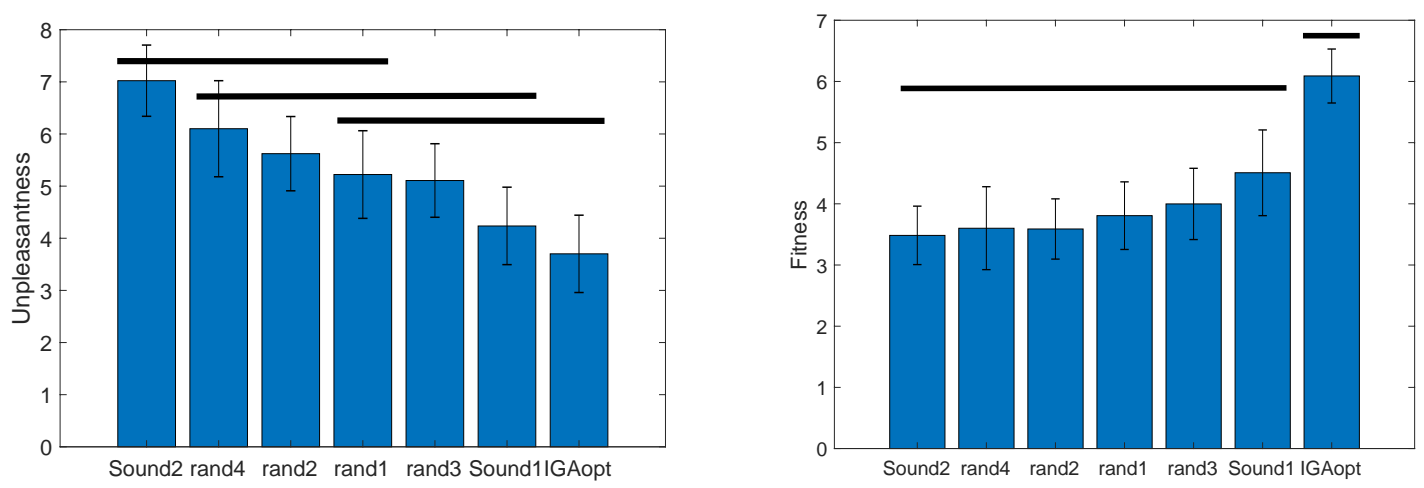

\begin{abstract}
Figure 7. Bar graph of the average value of the Unpleasantness (left) and Fitness (right) for the different EV sounds. Non significant differences between pairs of sounds ( $p>05)$ are linked with an horizontal line (paired t-test).
\end{abstract}

These results are average results for the whole panel. At the individual level, the IGA sounds got the best fitness for 7 participants out of 14. The IGA procedure is interesting to define convenient EV sounds, optimized for unpleasantness and detectability. It allows ones to uncover tradeoffs between detectability and unpleasantness.

We are of course aware that there is a bias in the evaluation process because the same experimental protocol is used for the optimization and for the evaluation. A different experiment should be proposed, why not in real environment, to prove with an independent experiment that the IGA process is efficient to design EV sounds. This will be conducted in future experiments. The positive results are nevertheless encouraging given the small number of studies on the interactive optimization of sounds. 


\section{CONCLUSION}

This paper presented an experiment aiming at designing EV sounds that are detectable but not unpleasant. An Interactive Genetic Algorithm was implemented in order to optimize the fitness of synthesized sounds. The designed experimental protocol provided a realistic assessment of the detection time. With hearing tests, and inclusions of the EV sounds in a background sound, assessments of the detection time and the unpleasantness of the sounds were provided. First results show the potential of the method: the IGA algorithm is effective for the design of efficient sounds. Comparisons between sound proposals showed that in average, the sound provided by the IGA was significantly more efficient. Several perspectives can be drawn for this project. We are of course aware that our study would need more participants to confirm these first encouraging results. A next stage of the project will be to explain the performances in detectability and unpleasantness with sound parameters (spectral or spectro-temporal). This will be important to be able to draw recommendations to a sound designer and to improve the design of sounds for EVs.

\section{REFERENCES}

Campillo-Davo, N. and Rassili, A. (2016), NVH Analysis Techniques for Design and Optimization of Hybrid and Electric Vehicles, Shaker Verlag Publications. ISBN 978-3-8440-4356-3

Gillibrand, A., Suffield, I., Vinamata, X., Williams, R. and Brückmann, A. (2011), “An Initial Study to Develop Appropriate Warning Sound for a Luxury Vehicle Using an Exterior Sound Simulator", SAE International, 2011-01-1727

Goldberg, D.E. (1989), Genetic Algorithms in search, optimization \& machine learning, Addison-Wesley Publishing Company.

Kim, H.S. and Cho, S.B. (2006), "Application of Interactive Genetic Algorithm to Fashion Design”, Engineering Design, Vol. 38, pp. 224-237.

Konet, H., Sato, M., Schiller, T., Christensen, A., Tabata, T. and Kanuma, T. (2011), "Development of Approaching Vehicle Sound for Pedestrians (VSP) for Quiet Electric Vehicles", SAE International, pp. 2011-01. -0928.

Kerber, S. and Fastl, H. (2008), "Prediction of perceptibility of vehicle exterior noise in background noise", Proceedings of DAGA' 08 conference, Dresden, Germany.

Lee, J.H. and Chang, M.L. (2010), "Stimulating designers' creativity based on a creative evolutionary system and collective intelligence in product design", International Journal of Industrial Ergonomics, Vol. 40, pp. 295-305.

Lee, S.K., Lee, S.M., Shin, T. and Han, M. (2017), "Objective evaluation of the sound quality of the warning sound of electric vehicles with a consideration of the masking effect: annoyance and detectability", International Journal of Automotive Technology, Vol. 18 No. 4, pp. 699-705. https://doi.org/10.1007/s12239-017-0069-6.

Misdariis, N., Gruson, A. and Susini, P. (2013), "Detectability study of warning signals in urban background noises: A first step for designing the sound of electric vehicles", Proc. Mtgs. Acoust., Vol. 19, pp. 040032. https://doi.org/10.1121/1.4799454.

Misdariis, N., Cera, A., Levallois, E. and Locqueteau, C. (2012), "Do electric cars have to make noise? An emblematic opportunity for designing sounds and soundscapes", Proc. of Acoustics 2012, Nantes, France.

Parizet, E., Ellermeier, W. and Robart, R. (2014), "Auditory warnings for electric vehicles: Detectability in normal-vision and visually-impaired listeners", Applied Acoustics, Vol. 86, pp. 50-58.

Petiot, J.-F., Kristensen, B.G. and Maier, A.M. (2013), "How should an electric vehicle sound? User and expert perception", Proceedings of IDETC/CIE 2013, Portland, Oregon, USA, August 4-7, 2013.

Poirson, E., Petiot, J.-F., Boivin, L. and Blumenthal, D. (2013), "Eliciting User Perceptions Using Assessment Tests Based on an Interactive Genetic Algorithm”, ASME. J. Mech. Des, Vol. 135 No. 3), 031004-031004-16.

Qian, L. and Ben-Arieh, D. (2009), "Joint pricing and platform configuration in product family design with genetic algorithm", Proceedings of IDETC/CIE 2009, San Diego, CA, USA.

Robart, R., Parizet, E., Chamard, J.C., Janssens, K. and Biancardi, F. (2013), "eVADER: A perceptual approach to finding minimum warning sound requirements for quiet cars", AIA-DAGA 2013 Conf. Acoustics, Vol. 1.

Rossignol, M., Lafay, G., Lagrange, M. and Misdariis, N. (2015), "SimScene: a web-based acoustic scenes simulator", 1st Web Audio Conference (WAC).

SenseLab Report (2011), White Paper on External Warning Sounds for Electric Cars. Available at: http://live.unece.org/fileadmin/DAM/trans/doc/2011/wp29grb/QRTV-06-04e.pdf.

Singh, S., Payne, S.R. and Jennings, P.A. (2014), "Toward a methodology for assessing electric vehicle exterior sounds", IEEE Trans. Intelligent Transportation Systems, Vol. 15 No. 4, pp. 1790-1800.

Wakefield, G.H., Van den Honert, C., Parkinson, W. and Lineaweaver, S. (2005), "Genetic Algorithms for Adaptive Psychophysical Procedures: Recipient-Directed Design of Speech-Processor MAPs", Ear and Hearing, Vol. 26 No. 4, pp. 57S-72S. 